\title{
ERRATUM
}

\section{ORTHODROME-LOXODROME CORRELATION BY THE MIDDLE LATITUDE RULE - ERRATUM}

\section{Miljenko Petrović}

doi:10.1017/S037346331300074X, Published by Cambridge University Press, 20 November 2013.

The publisher apologises for the errors in Paragraph 1 of the Introduction. Lines 6-10 of paragraph one should read as follows:

Finding the infinitesimal distance $d D_{O}$ that the vessel must cover in Great Circle sailing for the orthodromic course to change by a small value $d C_{O}$ illustrates the problem. Referring to Figure 1, from a spherical right angled triangle TVP with any two parts given any third can be found as follows:

And in Table 1, there are errors in Line 1 and Line 5. Lines 1 and 5 should read as follows:

Line 1

$5^{\circ} / 25^{\circ}-14^{\circ} 17 \cdot 6^{\prime} / 79^{\circ} 11 \cdot 2^{\prime} \quad 22^{\circ} 25 \cdot 3^{\prime} / 51^{\circ} 25 \cdot 1^{\prime} \quad 70 \cdot 7^{\circ} / 84 \cdot 2^{\circ} \quad 4702 \cdot 7^{\prime} / 4695 \cdot 1^{\prime} \quad 4685 \cdot 9^{\prime}$

Line 5

$45^{\circ} / 65^{\circ}-58^{\circ} 28 \cdot 0^{\prime} / 62^{\circ} 12 \cdot 3^{\prime} \quad 62^{\circ} 05 \cdot 6^{\prime} / 33^{\circ} 54 \cdot 3^{\prime} \quad 49 \cdot 2^{\circ} / 77 \cdot 0^{\circ} \quad 2361 \cdot 7^{\prime} / 2344 \cdot 6^{\prime} \quad 2323 \cdot 2^{\prime}$

The corrected Table is given below.

Table 1. Middle Latitude Rule (Sphere).

\begin{tabular}{lccccc}
\hline$\varphi_{\mathrm{T}} / \varphi_{\mathrm{V}}$ & $\lambda_{0} / \lambda_{\mathrm{V}}$ & $\varphi_{\mathrm{I}} / \lambda_{\mathrm{I}}$ & $\theta_{\mathrm{T}} / \theta_{\mathrm{I}}$ & $\mathrm{D}_{\mathrm{TLV}} / \mathrm{D}_{\mathrm{TIV}}$ & $\mathrm{D}_{\mathrm{GC}}$ \\
\hline $5^{\circ} / 25^{\circ}$ & $-14^{\circ} 17 \cdot 6^{\prime} / 79^{\circ} 11 \cdot 2^{\prime}$ & $22^{\circ} 25 \cdot 3^{\prime} / 51^{\circ} 25 \cdot 1^{\prime}$ & $70 \cdot 7^{\circ} / 84 \cdot 2^{\circ}$ & $4702 \cdot 7^{\prime} / 4695 \cdot 1^{\prime}$ & $4685 \cdot 9^{\prime}$ \\
$15^{\circ} / 35^{\circ}$ & $-33^{\circ} 24 \cdot 8^{\prime} / 67^{\circ} 30 \cdot 0^{\prime}$ & $32^{\circ} 11 \cdot 2^{\prime} / 41^{\circ} 30 \cdot 7^{\prime}$ & $65 \cdot 6^{\circ} / 82 \cdot 6^{\circ}$ & $3813 \cdot 8^{\prime} / 3803 \cdot 4^{\prime}$ & $3790 \cdot 6^{\prime}$ \\
$25^{\circ} / 45^{\circ}$ & $-46^{\circ} 02 \cdot 0^{\prime} / 62^{\circ} 12 \cdot 3^{\prime}$ & $42^{\circ} 05 \cdot 5^{\prime} / 36^{\circ} 48 \cdot 0^{\prime}$ & $60 \cdot 7^{\circ} / 81 \cdot 0^{\circ}$ & $3226 \cdot 4^{\prime} / 3213 \cdot 7^{\prime}$ & $3197 \cdot 8^{\prime}$ \\
$35^{\circ} / 55^{\circ}$ & $-54^{\circ} 22 \cdot 5^{\prime} / 60^{\circ} 38 \cdot 4^{\prime}$ & $52^{\circ} 03 \cdot 9^{\prime} / 34^{\circ} 34 \cdot 8^{\prime}$ & $55 \cdot 5^{\circ} / 79 \cdot 3^{\circ}$ & $2767 \cdot 0^{\prime} / 2752 \cdot 1^{\prime}$ & $2733 \cdot 4^{\prime}$ \\
$45^{\circ} / 65^{\circ}$ & $-58^{\circ} 28 \cdot 0^{\prime} / 62^{\circ} 12 \cdot 3^{\prime}$ & $62^{\circ} 05 \cdot 6^{\prime} / 33^{\circ} 54 \cdot 3^{\prime}$ & $49 \cdot 2^{\circ} / 77 \cdot 0^{\circ}$ & $2361 \cdot 7^{\prime} / 2344 \cdot 6^{\prime}$ & $2323 \cdot 2^{\prime}$ \\
\hline
\end{tabular}

\section{REFERENCE}

Miljenko Petrović. Orthodrome-Loxodrome Correlation by the Middle Latitude Rule. Journal of Navigation. Published by Cambridge University Press, 20 November 2013. doi:10.1017/ S037346331300074X. 